\title{
Polycystic ovary syndrome: clinical implication in perimenopause
}

\author{
Monika Lenart-Lipińska ${ }^{1,2}$, Beata Matyjaszek-Matuszek ${ }^{2}$, Ewa Woźniakowska ${ }^{3}$, Janusz Solski ${ }^{1}$, \\ Jerzy S. Tarach², Tomasz Paszkowski ${ }^{3}$
}

${ }^{1}$ Department of Laboratory Diagnostics, Medical University of Lublin, Poland

${ }^{2}$ Department of Endocrinology, Medical University of Lublin, Poland

${ }^{3} 3^{\text {rd }}$ Chair and Department of Gynecology, Medical University of Lublin, Poland

\begin{abstract}
Polycystic ovary syndrome (PCOS), a hyperandrogenic disorder, is the commonest endocrinopathy in premenopausal women. This syndrome is associated with fertility problems, clinical manifestations of hyperandrogenism and metabolic disturbances, particularly insulin resistance and obesity. There is a great body of evidence that patients with PCOS present multiple cardiovascular risk factors and cluster components of metabolic syndrome from early ages. The presence of comorbidities such as abdominal obesity, insulin resistance, type 2 diabetes, hypertension places these females at an increased risk of future cardiovascular events. However, the extent to which PCOS components are present in perimenopausal women and the degree to which PCOS increases various risk factors in addition to the known risk of the perimenopausal period have not been fully determined. The perimenopausal period per se is associated with weight gain and an increased cardiovascular risk, which may be additionally aggravated by the presence of metabolic disturbances connected with PCOS. The phenotype of PCOS may improve with aging and it is still uncertain whether the presence of PCOS significantly increases the cardiovascular risk later in women's life. Most recent data suggest that the prevalence of cardiovascular diseases and the related long-term consequences in females with PCOS seem to be lower than expected. This manuscript reviews long-term consequences of PCOS and considers their clinical implications in perimenopause.
\end{abstract}

Key words: PCOS, menopause, perimenopause, insulin resistance, cardiovascular risk.

\section{Introduction}

Polycystic ovary syndrome (PCOS) is considered to be the most common endocrine disorder in women and dependently on diagnostic criteria, accounts for approximately $10 \%$ of premenopausal women [1]. The clinical manifestation of PCOS is heterogeneous, varies widely between patients, but the diagnosis of the classic phenotype is mainly based on a triad of symptoms that include hyperandrogenism (either clinical or biochemical), oligoovulation or anovulation and the presence of polycystic ovaries in ultrasound examination [2]. The etiology of PCOS is multifactorial and still not fully understood [1]. The numerous research about its pathogenesis have shown that familial and genetic factors may cause predisposition to PCOS [3]. Data cover the potential fetal developmental programming to the molecular basis of adipose tissue dysfunction, insulin resistance, inflammation, oxidative stress and endothelial dysfunction [4]. However, the basic pathophysiologic defect in PCOS has not yet been established. Insulin resistance and subsequent hyperinsulinemia are major features of PCOS and occur in up to $80 \%$ of all affected females and in almost all obese women with PCOS [5]. It has been proven that insulin per se, through IGF1R signaling or through classical insulin receptor signaling, can enhance androgen secretion and induce development of polycystic ovaries. Furthermore, hyperinsulinemia inhibits hepatic production of sex hormone binding globulin (SHBG) and in that mechanism increases the level of free testosterone in serum. Additionally, increased androgen levels facilitate the release of free fatty acids from visceral fat tissue, what exacerbates insulin resistance [6]. Insulin resistance in PCOS is also associated with abdominal obesity and other components of metabolic syndrome. All these abnormalities place females with PCOS at an increased risk of cardiovascular disorders later in life [7].

\section{Diagnostic criteria}

Three sets of diagnostic classifications can be currently applied to the diagnosis of PCOS: the National Institutes of Health (NIH) criteria, the Rotterdam criteria and the Androgen Excess and PCOS Society 
criteria. All of these systems require the exclusion of other disorders, such as androgen producing tumors, congenital adrenal hyperplasia, Cushing's syndrome, thyroid dysfunction and hyperprolactinemia. According to NIH criteria, PCOS can be recognized if there is a combination of oligo- or anovulation and clinical or biochemical features of hyperandrogenism [8]. According to the Rotterdam criteria, PCOS is diagnosed in the presence of two out of the following symptoms: oligo- or anovulation, hyperandrogenism and the presence of polycystic ovaries [9]. The PCOS diagnosis made in accordance with the most recent criteria of the Androgen Excess and PCOS Society, needs confirming the presence of hyperandrogenism which is considered as central abnormality, with ovarian dysfunction [10].

\section{Polycystic ovary syndrome and perimenopausal period}

The diagnosis of PCOS in perimenopause can be difficult to establish. It has been reported that clinical and biochemical manifestations of PCOS change with age and the phenotype of PCOS in women in the perimenopausal period is uncertain and difficult to define [11]. Menopausal transition as a physiologic process occurs along with aging, and itself is associated with several hormonal and metabolic changes, what may be challenging to be distinguished from abnormalities connected with PCOS. Numerous studies confirm that in perimenopausal women there is increased prevalence of metabolic syndrome what is connected with observed weight gain, especially in abdominal fat tissue, hypertension and their clinical consequences in this population of females [12]. According to many observations, the classic phenotype of PCOS, connected with hyperandrogenism, oligoovulation and polycystic ovaries, ameliorates with aging [13]. That is why widely used criteria for diagnosis of PCOS may not be useful in perimenopause. The disturbances in ovulation are common among young women and menstrual cycles may become regular with age in women with PCOS [14]. Furthermore, ovarian volume and number of follicles decrease with age in both healthy controls and women with PCOS [15]. Additionally, hyperandrogenism considered as the hallmark of the syndrome, may partially resolve in the perimenopausal period in women with PCOS [16]. This phenomenon can be explained by ovarian and adrenal aging and decreased androgens production in that mechanism [13]. However, it has been reported that androgens excess in patients with PCOS persists after the menopausal transition and postmenopausal women with PCOS are exposed to higher adrenal and ovarian androgen levels than non-PCOS women [17]. Data published by Puurunen et al. revealed that enhanced ovarian androgen secretion along with un- favorable metabolic changes such as impaired glucose tolerance, chronic inflammation, observed in a group of premenopausal women with PCOS, persist after the menopausal transition [18]. For PCOS diagnosis in perimenopause some studies have relied on a previous history of oligoovulation, the presence of polycystic ovaries and current features of hyperandrogenism: hyperandrogenemia or hirsutism [19]. Other researchers additionally have taken into account elevated waist circumference and biochemical evidence of insulin resistance for diagnosis of PCOS in the perimenopausal period [20].

\section{Insulin resistance and metabolic syndrome}

Metabolic syndrome is an insulin-resistant state, which is defined as a constellation of common cardiovascular risk factors, including visceral obesity, impaired carbohydrate metabolism, dyslipidemia and hypertension. It is believed that metabolic syndrome may predict a higher risk of cardiovascular events than the sum of the risks imparted by separate components [21]. There is a great body of evidence that PCOS and metabolic syndrome appear to be interrelated disorders linked together by insulin resistance. Metabolic syndrome and underlying insulin resistance are the major features in women with PCOS, while females with metabolic syndrome may develop reproductive or endocrine disturbances of PCOS. What is interesting, insulin resistance, as well as central adiposity, affects not only obese but also lean women with PCOS, that is why the population of PCOS females are prone to develop metabolic syndrome independently of obesity per se [3-6]. Additionally, hyperandrogenism commonly present in PCOS, may exacerbate the metabolic disturbances via the aggravation of central adiposity and promotion of insulin resistance [22]. Data reveal that lean PCOS patients in comparison to lean control subjects, were found to display more significantly an atherogenic lipid profile and other additional cardiovascular risk factors, not included in the classic definition of metabolic syndrome, such as increased markers of low-grade inflammation, impaired fibrinolysis and oxidative stress [23-25]. It has been established that the incidence of metabolic syndrome increases substantially during perimenopause and after menopause [12]. What is interesting, Livadas et al. reported an increase in insulin resistance along with aging, but only in obese women with PCOS [26]. They found that insulin resistance was positively associated with BMI and androgens level. Knowing the fact that androgens decline with aging, not obese females with PCOS may present a better metabolic profile with time and PCOS may remain even a silent disorder [26]. This observation strongly confirms the need of implementation of lifestyle modification against weight gain, 
what may ameliorate adverse cardiometabolic profile in PCOS females.

\section{Polycystic ovary syndrome and cardiovascular risk}

According to many research, PCOS should be considered as a metabolic disorder with an increased risk of future type 2 diabetes and cardiovascular disease [27]. The results of clinical studies in females with PCOS, which focused on surrogate outcomes for cardiovascular risk, have confirmed the existence of metabolic risk factors in this population of patients, such as endothelial dysfunction, platelet dysfunction, increased low grade inflammation, increased coronary artery calcification, arterial stiffness and carotid intima-media thickness [23-25]. Shaw et al. confirmed an increased number of cardiovascular events and higher incidence of diabetes, obesity, metabolic syndrome and coronary artery disease in a group of postmenopausal women with PCOS in comparison to other postmenopausal females [20]. Another study revealed a positive correlation between cardiovascular disease and a number of features of PCOS in postmenopausal women, what led to the conclusion that PCOS increases the risk of cardiovascular events after menopause [19]. Such observations may indicate the increased long-term risk of cardiovascular morbidity and mortality in PCOS females and explain the need of a complete and periodical cardiometabolic evaluation with the aim of preventing future events. However, despite a significantly higher risk of cardiovascular disorders, such as hypertension, atherogenic lipid profile, obesity, diabetes in females with PCOS, some studies to date have brought contradictory findings and failed to establish increased cardiovascular mortality or morbidity $[28,29]$. In the small Swedish longterm, a prospective follow-up study of PCOS women in postmenopausal age, the risk of acquiring myocardial infarction, stroke or death caused by cardiovascular disease did not differ between the groups. Furthermore, total mortality during 21-year follow-up period was similar in comparison to the control group despite the higher prevalence of cardiovascular risk factors among patients with PCOS [30]. These findings may support the notion that traditional cardiovascular risk factors may not be fully applicable to a population of patients with PCOS and new markers are needed for better assessment and stratification of PCOS patients. In conclusion, although PCOS patients are considered to have an increased cardiovascular risk, recent available data suggest that the prevalence of cardiovascular diseases and the related cardiovascular risk in females with PCOS may be lower than expected [31]. However, further studies on a larger PCOS population with a long-time follow-up are required to investigate the impact of metabolic disturbances on cardiovascular events and overall mortality in PCOS patients.

\section{Polycystic ovary syndrome and risk of cancers}

In PCOS there are numerous potential mechanisms which could promote malignant disease, including the prolonged anovulatory status and associated imbalance between androgen and estrogen action, insulin resistance and secondary hyperinsulinemia [32, 33]. Many studies have been conducted in order to assess the risk of cancers in women with PCOS. Barry et al. performed a systematic review and meta-analysis of observational studies and concluded that women of all ages with PCOS are at increased risk of endometrial cancer [34]. The obtained data failed to confirm the increased risk of ovarian and breast cancer in PCOS patients [34]. Similarly, Haoula et al. found the same association between PCOS and a significantly increased risk of endometrial cancer in females with PCOS [35]. They analyzed data from comparative studies and concluded that women with PCOS were about three times more likely to develop endometrial cancer in comparison to patients not affected with PCOS. Research of others confirmed also a higher risk of ovarian cancer [36], although other studies have not supported this observation [28, 34]. Inconsistent findings also exist regarding the risk of breast cancer in women with PCOS $[28,37]$. More research is required to precisely define the overall cancer risk in PCOS population. However, current data indicate that in women with PCOS there is a significant cancer risk, at least for endometrium. This finding should facilitate proper screening strategies, lifestyle changes and metformin treatment as a primary intervention what may decrease the risk in PCOS population.

\section{Conclusions}

Polycystic ovary syndrome, the commonest endocrinopathy among premenopausal women, is also associated with metabolic dysfunction that may induce an increased cardiovascular risk, although long-term consequences and disease endpoints are not so far well established. Moreover, the phenotype of PCOS can vary widely throughout lifespan and may ameliorate with aging. The perimenopausal period is associated with weight gain and an increase in both total body fat and visceral fat tissue. The weight excess per se is associated with an increased cardiovascular risk, which may be additionally aggravated by the presence of metabolic disturbances connected with PCOS. However, it is still uncertain whether the presence of PCOS significantly increases the cardiovascular risk later in life. Most available data suggest that the prevalence of cardiovascular diseases and the related long-term consequences in 
females with PCOS seem to be lower than expected. But taking into account an increased risk of endometrial cancer and cardiovascular disease, especially in perimenopausal obese women, it should be emphasized that a diagnosis of PCOS, especially if accompanied by excess weight, must be followed by a complete and periodical cardiometabolic and gynecologic evaluation. There is a great body of evidence indicating that, when obesity is present, lifestyle intervention for the control of weight excess should be always considered, and if necessary combined with pharmacotherapy.

\section{Disclosure}

Authors report no conflicts of interest.

\section{References}

1. Goodarzi MO, Dumesic DA, Chazenbalk G, Azziz R. Polycystic ovary syndrome: etiology, pathogenesis and diagnosis. Nat Rev Endocrinol 2011; 7: 219-231.

2. Azziz R. PCOS: a diagnostic challenge. Reprod Biomed Online 2004; 8: 644-648.

3. Khan KA, Stas S, Kurukulasuriya LR. Polycystic ovarian syndrome. J Cardiometab Syndr 2006; 1: 125-130.

4. Dumesic DA, Abbott DH, Padmanabhan V. Polycystic ovary syndrome and its developmental origins. Rev Endocr Metab Disord 2007; 8: 127-141.

5. Carmina E, Lobo RA. Use of fasting blood to assess the prevalence of insulin resistance in women with polycystic ovary syndrome. Fertil Steril 2004; 82: 661-665.

6. Holte J. Disturbances in insulin secretion and sensitivity in women with polycystic ovary syndrome. Baillieres Clin Endocrinol Metab 1996; 10: 221-247.

7. Luque-Ramírez M, Escobar-Morreale HF. Polycystic ovary syndrome as a paradigm for prehypertension, prediabetes, and preobesity. Curr Hypertens Rep 2014; 16: 500.

8. Zawadski JK, Dunaif A. Diagnostic criteria for polycystic ovary syndrome: towards a rational approach. In: Dunaif AGJ, Haseltine F (eds.). Polycystic ovary syndrome. Blackwell Scientific, Boston 1992, pp. 377 384

9. Rotterdam ESHRE/ASRM-Sponsored PCOS Consensus Workshop Group. Revised 2003 consensus on diagnostic criteria and long-term health risks related to polycystic ovary syndrome. Fertil Steril 2004; 81: 19-25.

10. Azziz R, Carmina E, Dewailly D, et al.; Task Force on the Phenotype of the Polycystic Ovary Syndrome of The Androgen Excess and PCOS Society. The Androgen Excess and PCOS Society criteria for the polycystic ovary syndrome: the complete task force report. Fertil Steril 2009; 91: 456-488.

11. Hsu MI. Changes in the PCOS phenotype with age. Steroids 2013; 78: 761-766.

12. Lobo RA. Metabolic syndrome after menopause and the role of hormones. Maturitas 2008; 60: 10-18.

13. Brown ZA, Louwers YV, Fong SL, et al. The phenotype of polycystic ovary syndrome ameliorates with aging. Fertil Steril 2011; 96: 1259-1265.

14. Elting MW, Korsen TJ, Rekers-Mombarg LT, Schoemaker J. Women with polycystic ovary syndrome gain regular menstrual cycles when ageing. Hum Reprod 2000; 15: 24-28.

15. Alsamarai S, Adams JM, Murphy MK, et al. Criteria for polycystic ovarian morphology in polycystic ovary syndrome as a function of age. J Clin Endocrinol Metab 2009; 94: 4961-4970.

16. Winters SJ, Talbott E, Guzick DS, et al. Serum testosterone levels decrease in middle age in women with the polycystic ovary syndrome. Fertil Steril 2000; 73: 724-729.
17. Markopoulos MC, Rizos D, Valsamakis G, et al. Hyperandrogenism in women with polycystic ovary syndrome persists after menopause. J Clin Endocrinol Metab 2011; 96: 623-631.

18. Puurunen J, Piltonen T, Morin-Papunen L, et al. Unfavorable hormonal, metabolic, and inflammatory alterations persist after menopause in women with PCOS. J Clin Endocrinol Metab 2011; 96: 1827-1834.

19. Krentz AJ, von Mühlen D, Barrett-Connor E. Searching for polycystic ovary syndrome in postmenopausal women: evidence of a dose-effect association with prevalent cardiovascular disease. Menopause 2007; 14: 284-292.

20. Shaw LJ, Bairey Merz CN, Azziz R, et al. Postmenopausal women with a history of irregular menses and elevated androgen measurements at high risk for worsening cardiovascular event-free survival: results from the National Institutes of Health - National Heart, Lung, and Blood Institute sponsored Women's Ischemia Syndrome Evaluation. J Clin Endocrinol Metab 2008; 93: 1276-1284.

21. Kandaraki E, Christakou C, Diamanti-Kandarakis E. Metabolic syndrome and polycystic ovary syndrome... and vice versa. Arq Bras Endocrinol Metabol 2009; 53: 227-237.

22. Phillips GB, Jing T, Heymsfield SB. Does insulin resistance, visceral adiposity, or a sex hormone alteration underlie the metabolic syndrome? Studies in women. Metabolism 2008; 57: 838-844.

23. Conway GS, Agrawal R, Betteridge DJ, Jacobs HS. Risk factors for coronary artery disease in lean and obese women with the polycystic ovary syndrome. Clin Endocrinol (Oxf) 1992; 37: 119-125.

24. Christakou C, Diamanti-Kandarakis E. Structural, biochemical and nontraditional cardiovascular risk markers in PCOS. Curr Pharm Des 2013; 19: 5764-5774.

25. González F, Rote NS, Minium J, Kirwan JP. Reactive oxygen speciesinduced oxidative stress in the development of insulin resistance and hyperandrogenism in polycystic ovary syndrome. J Clin Endocrinol Metab 2006; 91: 336-340.

26. Livadas S, Kollias A, Panidis D, Diamanti-Kandarakis E. Diverse impacts of aging on insulin resistance in lean and obese women with polycystic ovary syndrome: evidence from 1345 women with the syndrome. Eur J Endocrinol 2014; 171: 301-309.

27. Guzick DS. Cardiovascular risk in PCOS. J Clin Endocrinol Metab 2004; 89: 3694-3695.

28. Pierpoint T, McKeigue PM, Isaacs AJ, et al. Mortality of women with polycystic ovary syndrome at long-term follow-up. J Clin Epidemiol 1998; 51: 581-586.

29. Wild S, Pierpoint T, McKeigue P, Jacobs H. Cardiovascular disease in women with polycystic ovary syndrome at long-term follow-up: a retrospective cohort study. Clin Endocrinol (Oxf) 2000; 52: 595-600.

30. Schmidt J, Landin-Wilhelmsen K, Brännström M, Dahlgren E. Cardiovascular disease and risk factors in PCOS women of postmenopausal age: a 21-year controlled follow-up study. J Clin Endocrinol Metab 2011; 96: 3794-3803.

31. Fauser BC, Bouchard P. Uncertainty remains in women with PCOS regarding the increased incidence of cardiovascular disease later in life, despite the indisputable presence of multiple cardiovascular risk factors at a young age. J Clin Endocrinol Metab 2011; 96: 3675-3677.

32. Genazzani AR, Gadducci A, Gambacciani M; International Menopause Society Expert Workshop. Controversial issues in climacteric medicine II. Hormone replacement therapy and cancer. Maturitas 2001; 40: 117-130.

33. Boyd DB. Insulin and cancer. Integr Cancer Ther 2003; 2: 315-329.

34. Barry JA, Azizia MM, Hardiman PJ. Risk of endometrial, ovarian and breast cancer in women with polycystic ovary syndrome: a systematic review and meta-analysis. Hum Reprod Update 2014; 20: 748-758.

35. Haoula Z, Salman M, Atiomo W. Evaluating the association between endometrial cancer and polycystic ovary syndrome. Hum Reprod 2012; 27: 1327-1331.

36. Chittenden BG, Fullerton G, Maheshwari A, Bhattacharya S. Polycystic ovary syndrome and the risk of gynaecological cancer: a systematic review. Reprod Biomed Online 2009; 19: 398-405.

37. Anderson KE, Sellers TA, Chen PL, et al. Association of Stein-Leventhal syndrome with the incidence of postmenopausal breast carcinoma in a large prospective study of women in lowa. Cancer 1997; 79: 494-499. 